\title{
Erratum to: The gut microbiota profile is associated with insulin action in humans
}

\author{
Matteo Serino · José Manuel Fernández-Real • Eduardo Garcia-Fuentes • \\ Maribel Queipo-Ortuño • José María Moreno-Navarrete · Álex Sánchez • \\ Rémy Burcelin · Francisco Tinahones
}

Published online: 29 August 2012

(C) Springer-Verlag 2012

\section{Erratum to: Acta Diabetol \\ DOI 10.1007/s00592-012-0410-5}

In the original publication of the article, the name of third author was published incorrectly. The correct name should read E. Garcia-Fuentes

The online version of the original article can be found under doi:10.1007/s00592-012-0410-5.

M. Serino $(\bowtie) \cdot$ R. Burcelin

Institut National de la Santé et de la Recherche Médicale (INSERM), Toulouse, France

e-mail: matteo.serino@inserm.fr

M. Serino $\cdot$ R. Burcelin

Unité Mixte de Recherche (UMR) 1048, Institut de Maladies Métaboliques et Cardiovasculaires (I2MC), Université Paul

Sabatier (UPS), 31432 Toulouse Cedex 4, France

J. M. Fernández-Real $(\varangle)$ · J. M. Moreno-Navarrete Department of Diabetes Endocrinology and Nutrition, Institut d'Investigació Biomédica de Girona, and CIBER Fisiopatologia Obesidad y Nutricion (CB06/03/010), Instituto de Salud Carlos III, Girona, Spain e-mail: jmfreal@idibgi.org

E. Garcia-Fuentes - M. Queipo-Ortuño · F. Tinahones Service of Endocrinology and Nutrition, Hospital Clinico Universitario Virgen de Victoria de Malaga and CIBEROBN (CB06/03/010), Instituto de Salud Carlos III, Madrid, Spain

\section{Á. Sánchez}

Statistics Department, Facultat de Biologia UB,

University of Barcelona, Avda Diagonal 645,

08028 Barcelona, Spain

\author{
Á. Sánchez \\ Institut de Recerca, Hospital Universitari Vall'Hebron, \\ Passeig Vall d'Hebron 112-119, 08035 Barcelona, Spain
}

